\title{
Detection of attention and meditation state-based brainwave system to control prosthetic arm
}

\author{
Ahmad Danial, Abdul Rahman², Hanim Hussin ${ }^{3}$ \\ 1,2,3 Faculty of Electrical Engineering, Universiti Teknologi Mara (UiTM), Selangor, Malaysia \\ ${ }^{3}$ Integrated Microelectronics System and Application Research Group, Universiti Teknologi Mara (UiTM), Selangor, \\ Malaysia
}

\begin{tabular}{l} 
Article Info \\
\hline Article history: \\
Received Oct 13, 2018 \\
Revised Dec 14, 2018 \\
Accepted Dec 28, 2018 \\
\hline
\end{tabular}

\section{Keywords:}

Brain-computer interface (BCI) Brain-machine interface (BMI) Electroencephalography (EEG) Neuroprosthetic NeuroSky

\begin{abstract}
Neurotechnology has led to the development of Brain-Computer Interfaces (BCIs) or Brain-Machine Interfaces (BMIs) which are devices that use brain transmission signal to operate. Electroencephalography (EEG) is one of the recent methods that could retrieve transmission signal of the brain from scalp safely. This paper will discuss the development of Neuroprosthetics limb by using patients' attention and meditation level to produce movement. The main objective of this project is to restore mobility of patients that have suffered from motor disabilities. This project is carried out by interfacing the data acquisition device which is NeuroSky Mindwaves Headset with the microcontroller to move the prosthetic arm as the output. Arduino Nano microcontroller acts as data processing and a controller to the arm as the output. The prosthetic arm is designed by using SOLIDWORKS software and fabricated by 3D printed. From this project, the user will be able to control the prosthetic arm ranging from rotating the hand to bending the fingers creating a grasp and release gesture.
\end{abstract}

Copyright $\odot 2019$ Institute of Advanced Engineering and Science. All rights reserved.

\section{Corresponding Author:}

Hanim Hussin,

Faculty of Electrical Engineering, Universiti Teknologi Mara (UiTM), 40450 Shah Alam, Selangor, Malaisia.

Email: hanimh@salam.uitm.edu.my

\section{INTRODUCTION}

The brain is the fastest supercomputer on earth, which also the command center of the human nervous system that controls everything in the human body including movement of a body. The building blocks of the brain produce brainwaves signals when neurons communicating with each other. Brainwaves are in the form of electrical pulses which measured in hertz $(\mathrm{Hz})$. Types of brainwaves are classified based on their frequencies which are Alpha, Beta, Theta, Delta, and Gamma. Each type of brainwave has its own functions and different signal wave [1]. Alpha waves are generated when human are in the state of relaxation or calmness and its frequency between 8 to $12 \mathrm{~Hz}$ and 7 to 13 pulses per second. Beta waves have the fastest waves between 12 to $38 \mathrm{~Hz}$ frequency and 13 to 60 pulses per second. It is usually generated during the human's normal state when they gain consciousness. For theta waves, they usually occur when human are in the deep meditation to act as a getaway in the learning process. The frequency range of theta waves is between $3 \mathrm{~Hz}$ to $5 \mathrm{~Hz}$. The brain can communicate and pass information both in conscious or unconscious mind. Delta is the slowest but has the loudest brainwaves between 0.5 to $3 \mathrm{~Hz}$. It is generated when a human takes a deep sleep and it emits between four cycles per second. Gamma waves have the largest frequency among all which is from 30 to $100 \mathrm{~Hz}$. Gamma waves are generated during active learning or in hyperactivity state. This state is the best time to retain information [2]. All of these waves can be obtained through four primary recording modalities which are Electroencephalography (EEG), Electrocorticography (ECoG), Local 
Field Potentials (LFPs), and Single-neuron action potential recordings (single units). The difference between all of these methods is where the electrode is placed whether on the scalp or in the head. Electroencephalography (EEG) is the safest method among all which electrodes are placed on the scalp to monitor electrical activity of the brain [3].

For a normal person, brainwaves are transmitted through the neuromuscular pathway to communicate with the external environment. In simple words, the electrical signal from the nervous system is transmitted to the muscle to produce movement. But for a person who suffers from motor disabilities, the signal from the brain cannot reach that far and may only settle for less. According to the statistic updated on June 2011 by Jabatan Kebajikan Masyarakat Malaysia, 115,000 Malaysian were registered with physical disabilities [4]. Physically disability usually refers to the loss of limb, deformed limbs at birth or paralysis of the limb. These people usually have limited movements. They will need help from others or from assistive technologies to survive their daily life. There are thousands of assistive products available for these people. Prosthetic arm is one of the assistive products available in the market nowadays. These artificial devices can be worn or surgically implanted to replace the diseased or missing limb. Several types of the prosthetic arm are the cosmetic arm, functional arm or both. Cosmetic prosthetic arm usually looks like a real human arm, but just a replica that cannot be moved. On the other hand, the functional prosthetic arm can flexibly work, but looks abnormal or robot like. Brain-Computer Interfaces (BCIs) or also known as Brain-Machine Interfaces (BMIs) are not new things to this world. For the past 40 years, researchers have seen the potential of these technologies and they have been trying to convert thought into action [5].

This project is developed to build a portable and lightweight, functional cosmetic prosthetic arm that can be controlled wirelessly through a wearable EEG Headset by using attention and meditation state of the users. The main objective of this project is to develop a Brain-Machine Interface device that could restore motor disabilities. The most significant impact of this project is to those disabled people who suffered from injured or loss of arm, deformed arm since birth or paralysed person who can use their brain to get help from a prosthetic arm. This prosthetic arm will ease paralysed people and amputees' daily life as a portable assistive device.

Recent studies have proven that neural interface systems have a big potential in restoring mobility of patients who suffered from motor disabilities as assistive devices $[6,7,8,9,10,13]$. This neural interface system used data from cortical activity recorded by several types of data acquisition devices. A previous study by Schwartz et al. [3] discovered several methods in retrieving data from the brain. They are classified based on the distance between the electrode and the cortex of brain as shown in Figure 1.

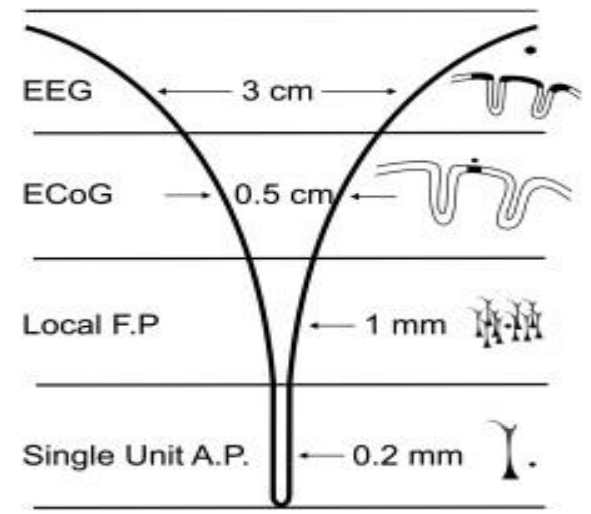

Figure 1. Comparisons of the four primary data acquisition of brain activities [3]

EEG electrodes located approximately 3 centimeters from the surface of the cortex. It is the safest way, but the large distances between the electrode and cortical surface makes it difficult to obtain the signal. Compared to EEG, ECoG obtains a finer signal because it lies approximately on the cortical surface. For local field potentials and single-unit action potentials, both are recorded in the brain parenchyma. All of these methods need surgery except EEG and every method has their pros and cons. Researchers have proven that every patient can use one of those methods as long as their brain are functioning. Even a paralyzed person's cerebral cortical motor areas become active when trying to move his/her body part [6, 8, 9, 11, and 12]. 


\section{RESEARCH METHOD}

This project is divided into several parts which are the design and development of the hardware and system This project is divided into several parts which are the design and development of the hardware and system and another is on the software. The design of the prosthetic arm and fabrication of the prototype are the main components in the design and development of the hardware. The system is designed to receive signals from the brain to move the arm. In the software part, a microcontroller is programmed to read the attention and meditation level from the sensors as the input and then rotate the servo motors as the output. The overview of the project implementation is shown in Figure 2.

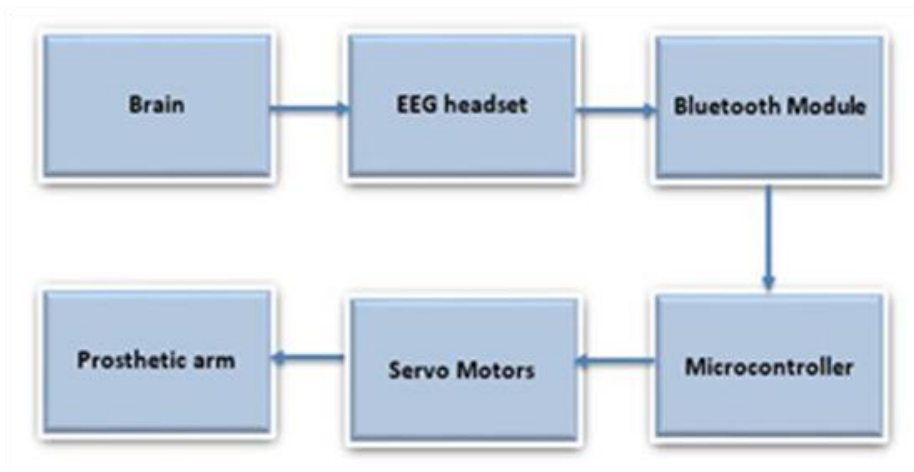

Figure 2. Overview of project implementation

\subsection{Hardware design}

The first phase in hardware design and development is designing the prosthetic arm using SOLIDWORKS software. The hand is designed based on a few pieces of research made to mimic a real human hand. The final design of the hand is shown in Figure 3. The design made is simulated using the same software to make sure the prosthetic arm is fully functional, and the hand can hold various types of shapes such as cylindrical shape, baseball's ball, and tiny things. Some aspects are considered in designing this prosthetic arm such as weight, size, and functionality. The last phase of hardware development is a fabrication of the arm using the 3D printer. The material used to fabricate the prosthetic arm is a biodegradable polymer, PLA that stands for Polylactic acid which is harder than ABS, Acrylonitrile Butadiene Styrene.
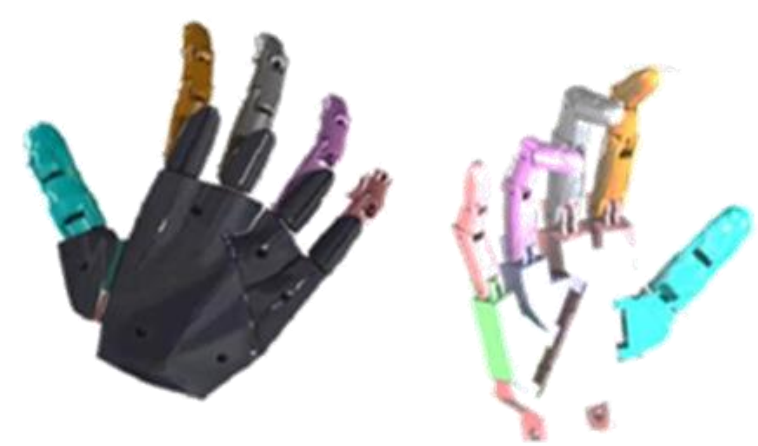

Figure 3. Final design of hand using SOLIDWORKS software

\subsection{System Architecture}

In developing and designing the system for this project, wearable wireless EEG headset, Mindwave from NeuroSky is used as the brain sensors to detect the brain's electrical activity. The electrical pulses are then converted into a signal and after that transferred to the microcontroller wirelessly through Bluetooth. Bluetooth module, HC-05 is connected to the microcontroller, Arduino to receive the signal transmitted from Mindwave Headset. Arduino microcontroller then interprets and process the signal received and transferred it 
to servo motors that are connected to strings in the prosthetic arm to create movement. The block diagram of the system is shown in Figure 4.

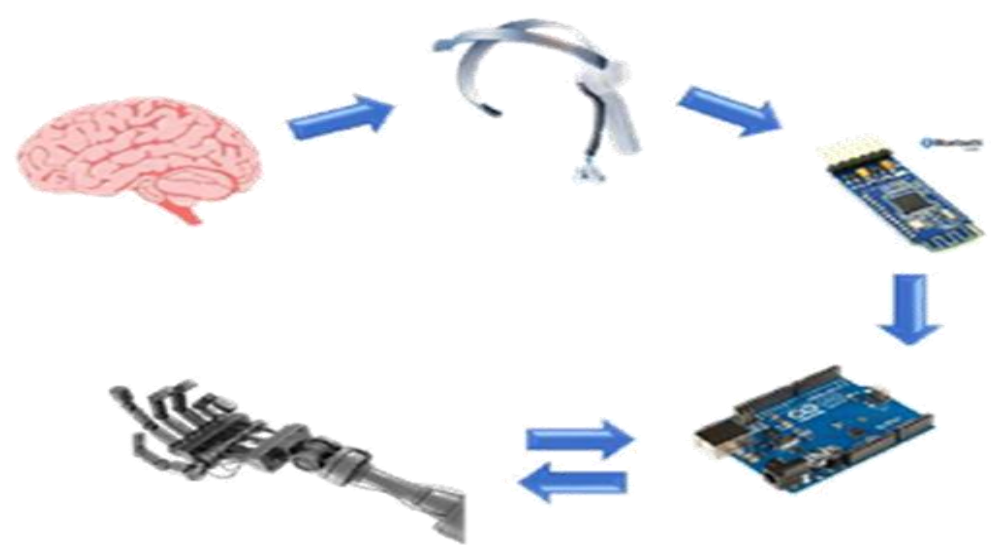

Figure 4. Block diagram of the system

\subsection{System Verification}

Figure 5 shows the setup for the Bluetooth module HC-05 to connect to EEG sensor and microcontroller board. LEDs are used as the substitution of the servo motor for verification purpose.

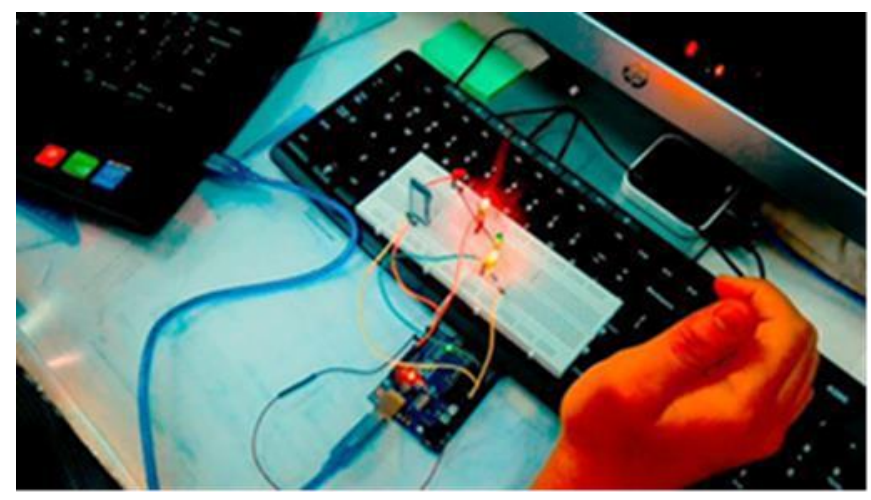

Figure 5. Testing setup to check the functionality of the system

\subsection{Software Implementation}

HyperTerminal is used to execute AT mode of the HC-05 in order to change the properties of the HC-05 Bluetooth module. This step is important to ensure the HC-05 will only pair with the headset. Deeper into the software part, the coding was made to detect the signal from the headset as the input to the microcontroller. The variables that were being focused are the strength in connection, attention level, meditation level and duration of the last data executed.

Attention levels are programmed to rotate the servo motors to pull the string in the prototype, so the hand will grasp while meditation levels are used to rotate the servo motors to rotate the hand. The software flowchart is shown in Figure 6. For this work, the result discussed is based on preliminary data upon signal detection and based on the wrist movement. Further work will be conducted to check the functionality for human application. 


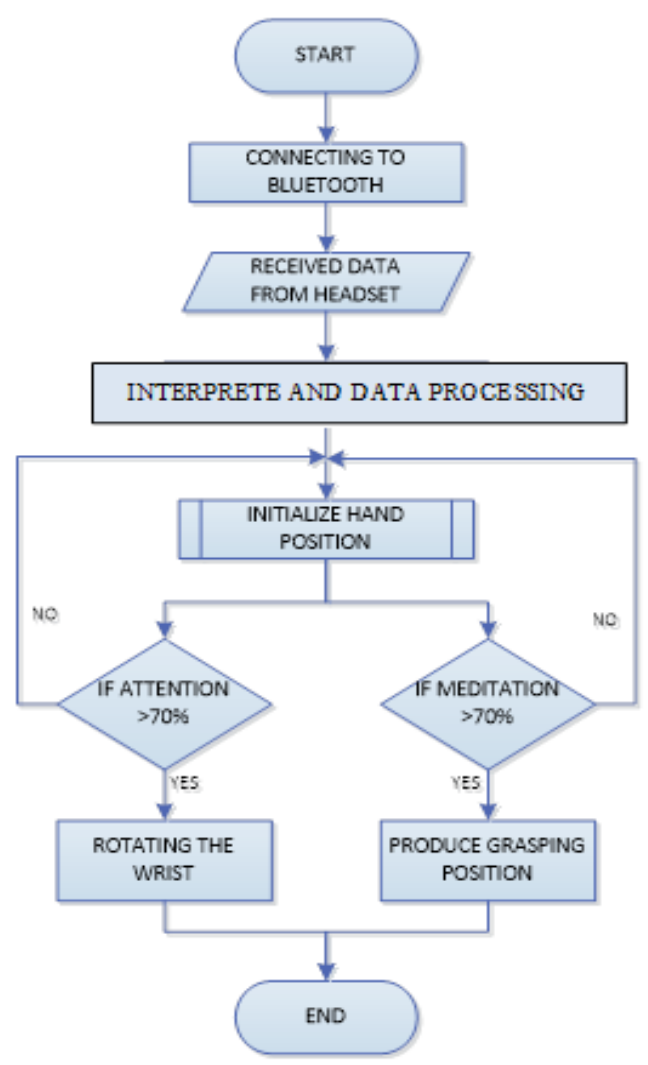

Figure 6. Software Flowchart of the system

\section{RESULTS AND ANALYSIS}

Figure 7(a) shows the graph of attention and meditation versus time in second when the user tries to get focused. At high levels of attention ( $70 \%$ and above), the grasping gesture would be produced as shown in Figure 7(b). If the attention level is below 70\%, it will release the grasp. The reason for setting the movement at the level of $70 \%$ is because it is quite difficult to achieve a definite $100 \%$ or $90 \%$ even. Besides, even if the user manages to reach $100 \%$ of attention level, it is near to impossible to maintain it. $70 \%$ is the average value of attention that indicates the user is about to get focused.

For meditation level, the same concept as the attention is used, but the difference lies only in the movement produced. Figure 8(a) shows the graph when the user tries to meditate. In this case, the high level of meditation ( $70 \%$ and above), would rotate the wrist. Figure $8(\mathrm{~b})$ show the initial condition of the hand and after rotating the wrist.

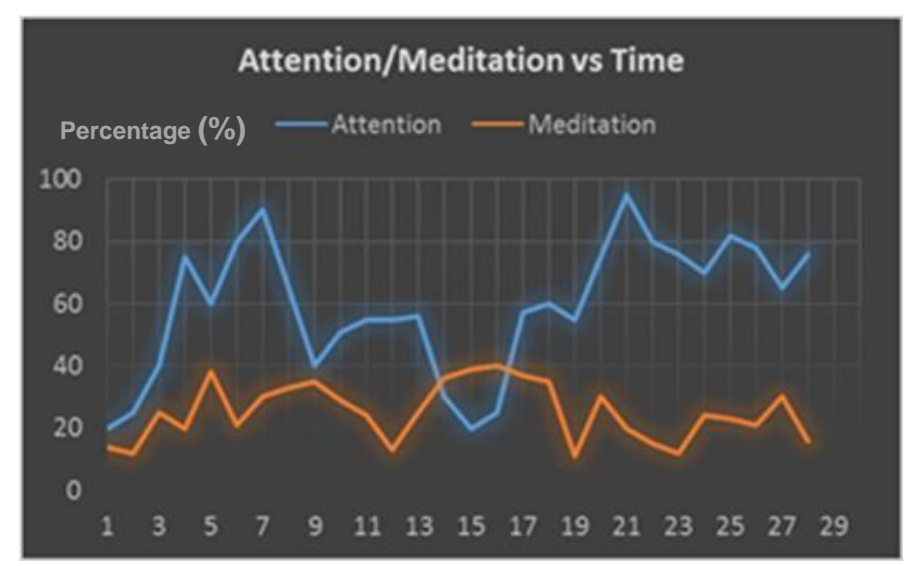

Figure 7(a). Graph of Attention and Meditation level (\%) versus Time(s) 

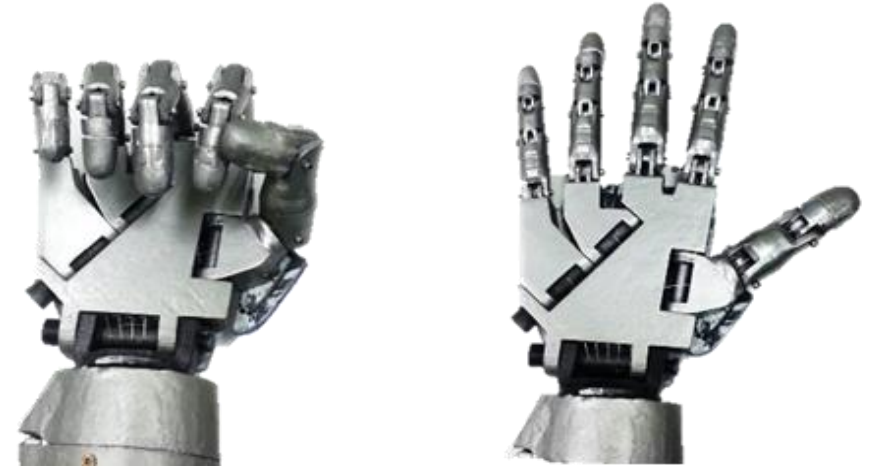

Figure 7(b). Grasping and release gesture of the hand

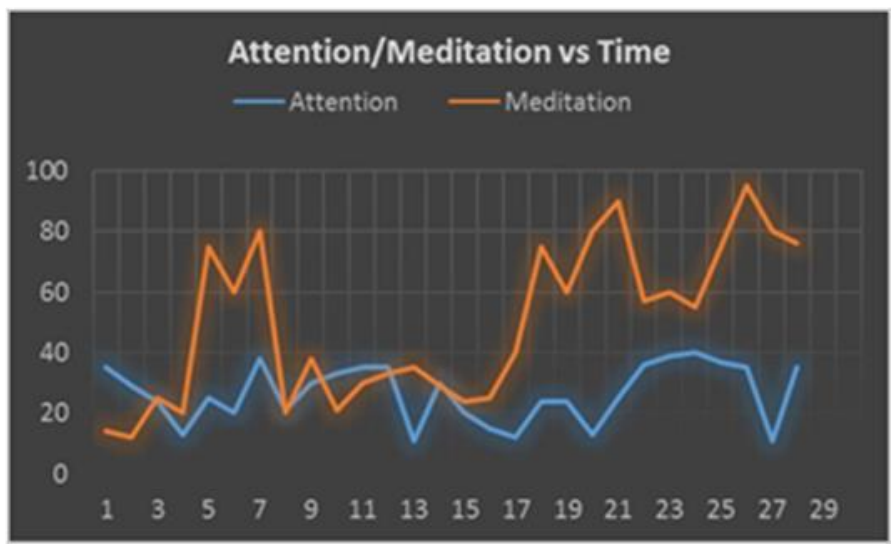

Figure 8(a). Graph of Attention and Meditation level (\%) versus Time(s)
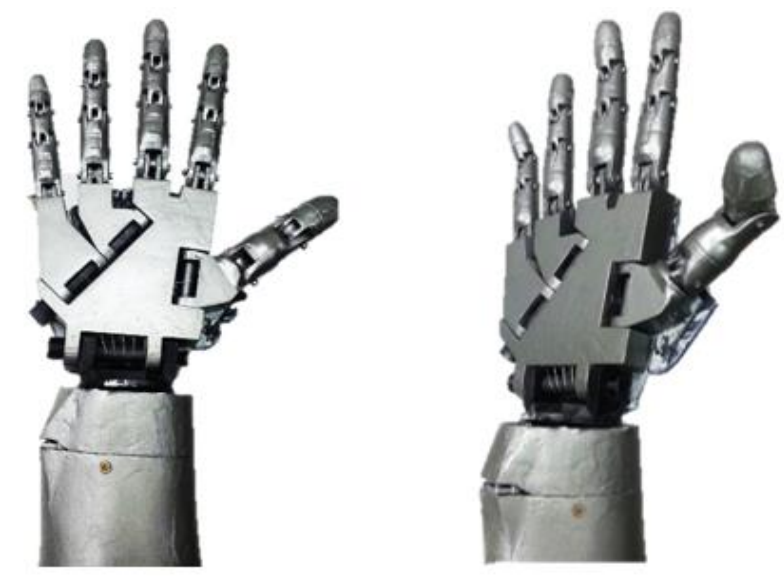

Figure 8(b). Initial condition of hand and after rotating wrist

\section{CONCLUSION}

The common problem that researcher faced in BCIs project is cost. By using a low-cost single electrode EEG headset like NeuroSky Mindwave for data acquisition is the limitation of BCIs projects. In a nutshell, this project is successful to control prosthetic arm based on attention and meditation state-based brainwave system. This project has the potential to give people who suffered from motor disabilities feel closer to normal functionality back. It is believed to give those disable hopes and sense of touch again ease their daily activities. For future improvements, it is better to use a multiple electrode EEG Headset such as 
Emotiv EPOC although it will cost some money. By using multiple electrode EEG headsets, the movement and gesture of the hand could be more complex and variety. Other than that, pressure sensors could be added at the fingertips to extend the force of grasping. Furthermore, the mechanism of hand can be added to bend the hand.

\section{REFERENCES}

[1] Brain Works (2015) What are Brain Waves. Retrieved from http://www.brainworksneurotherapy.com/what-arebrainwaves.

[2] Dr Hugo H. (2015) Brainwaves. Retrieved from http://www.doctorhugo.org/brainwaves/brainwaves.html.

[3] Andrew B. Schwartz,X. Tracy Cui,Douglas J. Weber,Daniel W. Moran, "Brain-Controlled Interfaces: Movement Restoration with Neural Prosthetics”, Neuron: Elsevier, Volume 52, Issue 1, 5 October 2006, Pages 205-220.

[4] Utusan Malaysia (2011) JKM kemas kini pendaftaran OKU. Retrieved from http://ww1.utusan.com.my/utusan/info.asp?y=2011\&dt=1103\&sec=Timur\&pg=wt_02.htm.

[5] Gerhard M. Friehs, MD; Vasilios A. Zerris, MD; Catherine L. Ojakangas, PhD;Mathew R. Fellows, BA; John P. Donoghue, $\mathrm{PhD}$ "Brain-Machine and Brain-Computer Interfaces" in press.

[6] Donoghue, J. P. "Bridging the brain to the world: a perspective on neural interface systems". (2008) Neuron 60, 511-521.

[7] Gilja, V. et al. "Challenges and opportunities for next-generation intra-cortically based neural prostheses". (2011) IEEE Trans. Biomed. Eng. 58, 1891-1899.

[8] Schwartz, A. B., Cui, X. T., Weber, D. J. \& Moran, D. W. "Brain-controlled interfaces: movement restoration with neural prosthetics". Neuron 52, 205-220 (2006).

[9] Nicolelis, M. A. L. \& Lebedev, M. A. "Principles of neural ensemble physiology underlying the operation of brainmachine interfaces". Nature Rev. Neurosci. 10, 530-540 (2009).

[10] Green, A. M. \& Kalaska, J. F. "Learning to move machines with the mind". Trends Neurosci.34, 61-75 (2011).

[11] J.R. Wolpaw, "Brain-computer interfaces as new brain output pathways", J. Physiol., 579 (2007), pp. 613-619.

[12] N.G. Hatsopoulos, J.P. Donoghue, "The science of neural interface systems",Annu. Rev. Neurosci., 32 (2009), pp. 249-266.

[13] Leigh R. Hochberg, Daniel Bacher, Beata Jarosiewicz, Nicolas Y. Masse, John D. Simeral, Joern Vogel, Sami Haddadin, Jie Liu, Sydney S. Cash, Patrick van der Smagt \& John P. Donoghue "Reach and grasp by people with tetraplegia using a neurally controlled robotic arm" in press. 